\title{
Automation of water meter billing process based on dig- ital image processing approach
}

\author{
Sudipto Chaki ${ }^{1 *}$, Dipu Akter Shila ${ }^{2}$, Nafsin Jahan ${ }^{3}$ \\ 1, 2, 3 Department of Computer Science \& Engineering, Bangladesh University of Business \& Technology, \\ Dhaka, Bangladesh
}

\author{
Keywords \\ Horizontal \& vertical projection \\ Hough transform \\ Correlation coefficient \\ Otsu threshold \\ Masked image \\ Projection profile
}

Received: 8 May 2019

Accepted: 10 July 2019

Published: 31 October 2019

\begin{abstract}
Automatic water meter reading recognition is the key feature of this paper from different environmental conditions. To do so, we have proposed a real-time automatic water meter reading recognition model in this research paper. Initially, from the meter image, the largest circular blob is extracted by creating a mask. As the water meter reading region can be tilted at a certain angle, the Hough transform is used to detect and correct the tilted angle. After tilt correction, to find out the circular meter region, the image masking technique is used following the largest blob finding technique. The corrected RGB meter image is converted to a grayscale image to reduce the computational cost for further processing. To improve the quality of the grayscale image, global histogram equalization is used. Using the Otsu threshold method, a gray image is converted to the binary image automatically. We have overcome the challenges of clipping the region of interest (ROI) extraction region. Applying horizontal and vertical projection, the region of interest (reading region) is extracted. At this point, a morphological operation has been implemented to reduce noise. To segment the digit image, a vertical projection algorithm is used. These segmented digits are then matched with template digit images following the correlation coefficient values. This work will overcome the problem of traditional water billing procedures and will be helpful to run a central water billing management.
\end{abstract}

(C) 2019 The Author(s). Published by TAF Publishing.

\section{INTRODUCTION}

Water is not only a vital element for human presence but also crucial for every animal to persist on the earth. But, day-by-day, lack of fresh water, and abuse is a threat to the current civilization. The main reasons for water deficit are population growth, acute droughts, overexploitation of groundwater, and uneven distribution of water resources. In recent years, the proper water distribution process has become a challenging job in Bangladesh because of corruption in this sector. So, we have considered the above cases and tried to find out a solution to solve this big problem. Some researchers have worked on this issue to find a suitable solution. Such a common type of advancement is using digital image processing systems. The automatic meter reading is the reading, transmission, storage, and processing of data exhibited on water meters through technolo- gies. The manual water meter reading process is erroneous and may fail sometimes and has several limitations like lowefficiency rate, shortage of manpower, etc. The improvement of an automatic water meter reading process has efficiently solved several problems caused by manual reading. It can keep track of the evidence of reading images furthermore. By recognizing the readings automatically from meter images, it can evaluate readings, generate bills, identify excessive water uses, diminish human errors, and reduce the bill processing cost. To acquire errorless reading, reading recognition, reading region detection from the water meter, researchers have demonstrated many efficient and effective methods.

Such as, in [1], the authors proposed a pre-processing technology that read water meter images automatically where Fourier transformation was used for detecting the edges.

\footnotetext{
${ }^{*}$ Corresponding author: Sudipto Chaki

†email: sudiptochakibd@gmail.com
} 
This method filters the effective region to edge detection process and then applies mathematical morphology operation. But, this technique only extracts the region of the print wheel effectively. No further detection or recognition work was performed. The authors in [2], proposed a simple image processing approach that used simple image processing algorithms and a Digital Signal Processor (DSP). The meter image has been captured from a fixed distance for better recognition purposes.

The horizontal and vertical pattern is generated in $[3,4]$ to segment each digit which is collected from the digit images. Here, the threshold values of $\mathrm{Cb}$ and $\mathrm{Cr}$ are used in terms of extracting the expected ROI. For recognition purposes, a customized horizontal and vertical pattern was used. A systematical process is introduced in $[5,6]$, to reduce the drawbacks of traditional electric meter detection and recognition process. This method uses the SVM which is a supervised learning-based classifier to recognize each digit from the images. The input image dataset is collected from realtime camera recordings.

Lamiaa A. Elrefaei et al. [7] proposed a digital image processing approach to recognize the electric wattmeter readings. Here, input datasets are sent through three main stages: first, it preprocesses the image, then segments each digit, and finally recognizes by comparing it with necessary templates. An automatic electric meter reading system is represented in [8], which consists of three tires with several reading terminals at the bottom, area concentrators, and the main computer for control and server in the middle and the main computer of the electrical administrative department at the top. In [9], Automatic Meter Recognition (AMR) is used where input meter images are collected through a CMOS image sensor. The wireless communication module is used to maintain a centralized server management system real-time meter recognition process.

K. Parthiban et al. proposed a technique in [10], where they compared their experimental results with the Sobel operator in terms of performance analysis. GSM interface module was applied in a wireless meter recognition network in [11]. A CCD (charge-coupled device) camera and a hierarchical computer system with pipelined image processors are developed for vehicle license numbers recognition in real-time [12]. In [13], the authors proposed an Optical Character Recognition (OCR) based method to detect LED/LCD characters in real-time. The system is applied both on a desktop and a smartphone.

\section{OUR PROPOSED METHODOLOGY}

Our proposed methodology can be classified into three steps: to identify the ROI from the water meter image; segmentation the image into an individual digit image and the recognition of each digit by matching with the proper pattern. The whole methodology is illustrated in Figure 1.

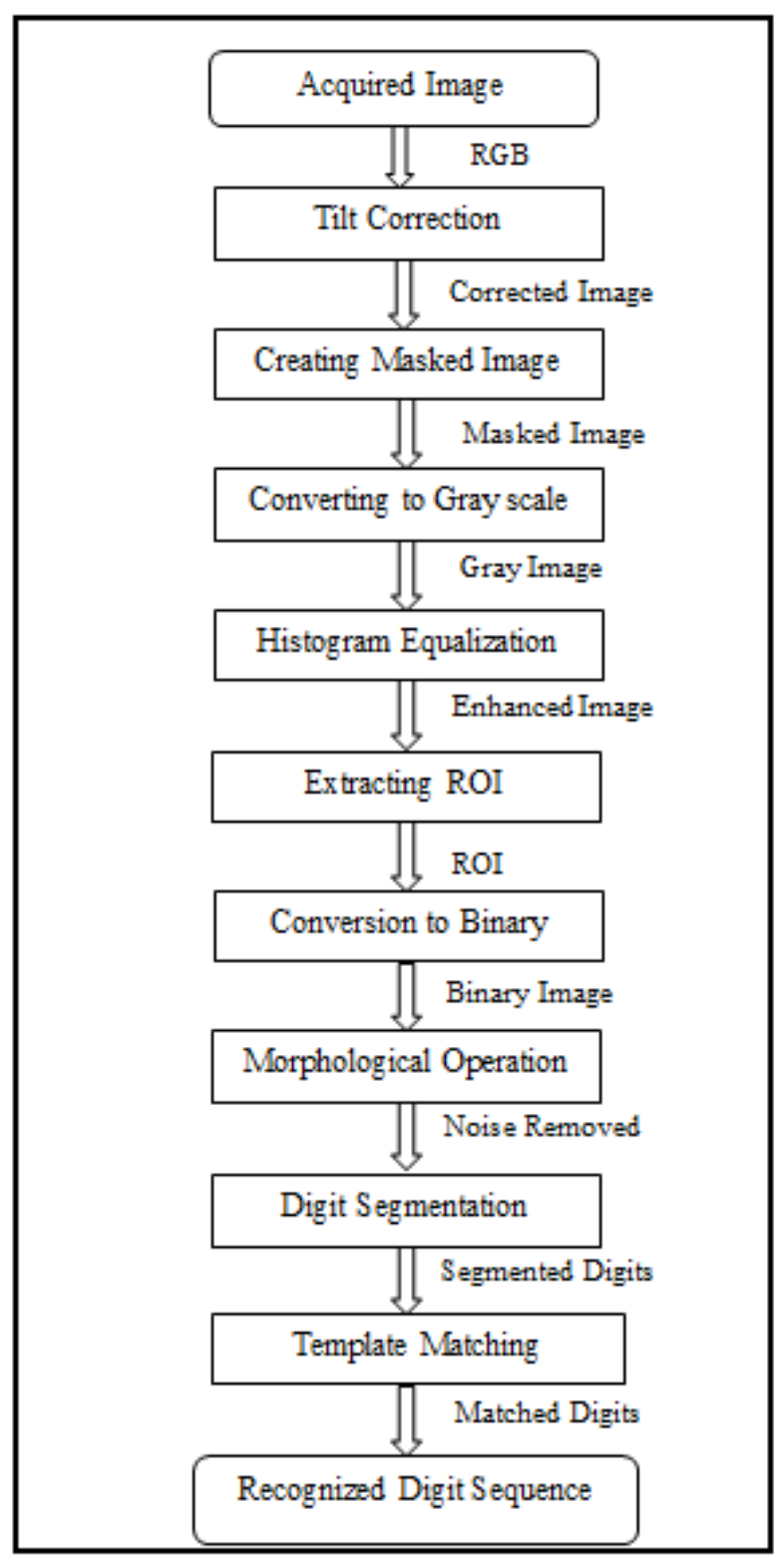

Fig. 1. The proposed framework on automation of the water meter billing process

\section{A. Image Acquisition and Preprocessing}

We have collected our water meter dataset by using smartphones. Then, all the captured images are fed into the automated detection and recognition system. The captured meter images are RGB images. So, the computation cost would be high. To progress to the next stages, the images have been converted to different color models to reduce the com- 
putational cost.

The preprocessing stage involves two basic steps. One is resizing and another is image enhancement. As the water meter can be located at different environmental conditions, we need to perform some pre-processing steps to get the result with the highest accuracy. The collected images are of different sizes so that processing can be difficult. To lift from this problem, the input images are resized into $(512 * 512)$.

\section{B. Tilt Correction}

The captured meter images can be tilted at a certain angle. Moreover, retrieving data from tilted images could be erroneous. To overcome this problem, we have implemented the Hough transformation algorithm in our datasets. It is a very widely used algorithm to find aligned points in images that create a line. A Fourier transformation based tilt correction technique has been used in [1]. Here, we have implemented the Hough Transformation algorithm as it gives more accuracy than the Fourier based method. In [2], the authors did not propose any tilt correction algorithm. But, in our proposed method, we have implemented a tilt correction algorithm to solve the issue. Here, we are providing some intuitions about the Hough transformation based tilt correction technique.

A line can be represented by the following mathematical representation shown in (1) where $\rho$ is the perpendicular distance to the origin of the line and $\theta$ is the angle formed by this perpendicular line and horizontal axis. There can be more than one single line in actual meter images. So, we need to map these points from meter space to Hough transfomation space by transfoming $(x, y)$ points to $(\theta, \rho)$ plane. Then the single points from a line will be intersected at the same point in Hough space shownin Figure 2 .

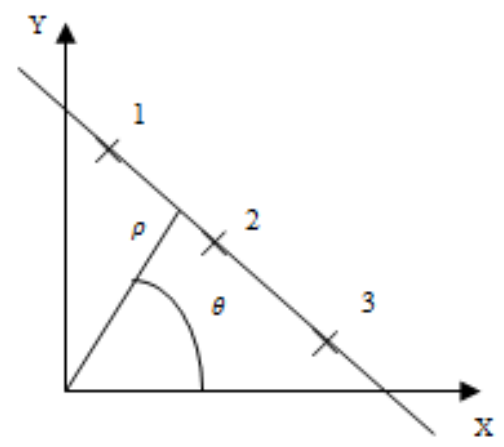

Fig. 2. Coordinate plane of an equation

$$
\rho=x \cos (\theta)+y \sin (\theta)
$$

After finding out the tilted angle, we need to correct the tilted angle. That's why we have implemented the image ro- tation technique. To correct the angle, we have performed an inverse transformation of every destination pixels. The following mathematical formulas have been implemented in the MATLAB environment shown in (2), and (3).

$$
\begin{aligned}
& x_{1}=r \cos (\alpha-\theta)=x_{0} \cos (\theta)+y_{0} \sin (\theta) \\
& y_{1}=r \sin (\alpha-\theta)=-x_{0} \sin (\theta)+y_{0} \cos (\theta)
\end{aligned}
$$

\section{Creating Mask}

To extract the largest circular blob, firstly, convert the RGB enhanced image into HSI or HSV color model. In the HSV color model, there are three channels just like RGB has. In Red, Green, and Blue (RGB), it has three color channels . On the contrary, the HSV color model, it has three color channels (Hue, Saturation, and Intensity). Here, we have used the intensity channel to get the threshold of the image. Using the largest blob finding technique, the circular largest blob is extracted. As the circular region is white, intensity channels help to threshold the blob area. Connected component labeling also called connected component analysis is an algorithmic use of chart hypothesis, where subsets of associated segments are interestingly named dependent on a given heuristic. Associated segment marking is utilized in computer vision to recognize associated districts in twofold advanced images. The whole process is illustrated in Figure 3.

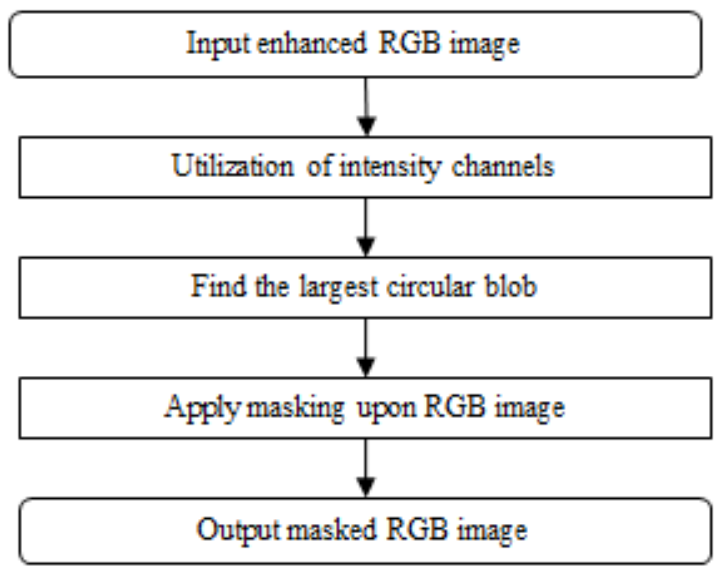

Fig. 3. Creating a masked image

\section{Converting to Grayscale Image}

In the RGB image, there will be $2^{\wedge} 8$ combinations of each color which makes it difficult to process information from this huge color combination of $\left(2^{\wedge} 24=16777216\right)$. On the other hand, in the grayscale image, there will be only $\left(2^{\wedge} 8=255\right)$ combinations of color. So, the processing is easier. Required to reduce the computational cost, the grayscale image would be a great option. It only contains 
one channel. It has only 8 bit to represent the color information.

\section{E. Histogram Equalization}

Histogram Equalization is the process that is involved with the enhancement of the given input images. There may be dust upon the surface on the meter images because the meter images are taken from environmental conditions. That's why we use histogram equalization. There are two types of histogram equalization to enhance the quality of the meter images: local histogram equalization and global histogram equalization. We use global histogram equalization in our project. Global histogram equalization improves the contrast of the image. It also obtains the uniform distribution of image intensity.

To enhance the given images, we need to find out the intensity levels of each image and then, need to set up the desired level. In the global histogram equalization procedure, all the same intensity of the pixels will be replaced by a cer- tain fixed value. That is why it is called a global histogram equalization. Besides, in local histogram equalization, we need to consider a frame size. Then, for each frame, the middle of the matrix value will be updated in the same manner. The reason behind the use of global histogram equalization is; it is faster and complexity is lower than local histogram equalization. By using the global technique, the image histogram is uniformly distributed for each level of intensities. Global histogram equalization involves the following steps in Figure 3.

\section{F. Extracting Region of Interest}

The main challenge of this project is to extract the ROI (region of interest) automatically from the meter image. As we only need the reading portion, it is required to extract that region and place the result in the next stage. In my project, we have used the horizontal and vertical projection histogram as the reference for extracting the ROI automatically.

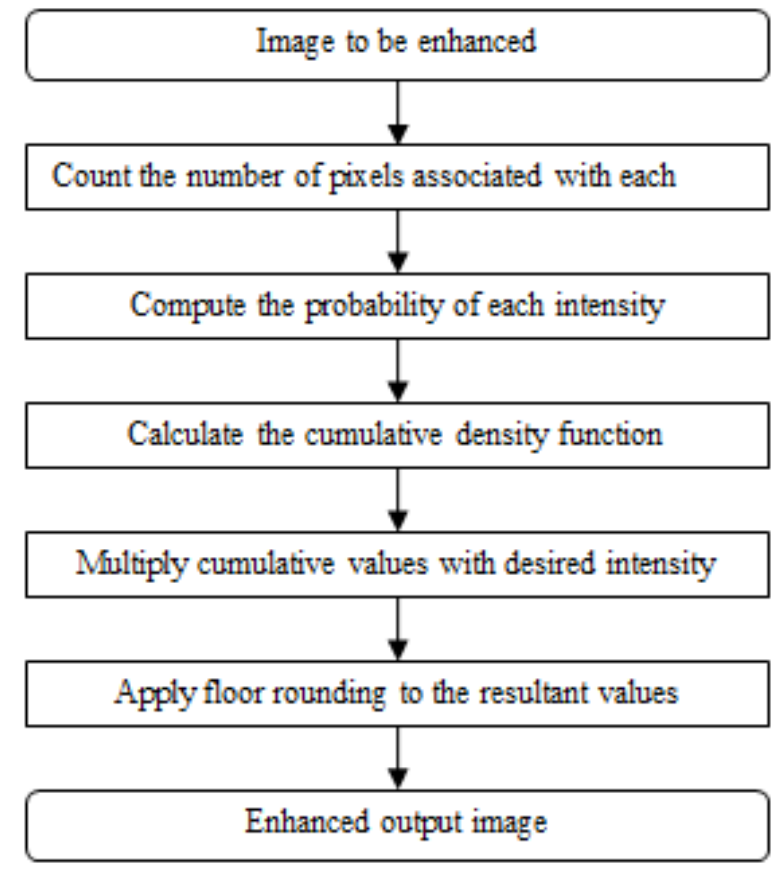

Fig. 4. Global histogram equalization

In [3], the region of interest was selected based on the $\mathrm{Cb}$ (the chromaticity of blue) and Cr (chromaticity of red) values. It was possible because the digital electric meter background is illuminated mostly by green color. But, in the case of mine, the water meter reading region is not illuminated. To extract the ROI effectively, at first, we have considered a horizontal projection profile which is shown in Figure 3. When the histogram is drawn, finding the third and fourth most significant peaks by sorting the values, the horizon- tal region can be clipped automatically using the peak information. The first and second most significant peaks are avoided because those are due to having a constant white circular region. By analyzing these peaks, we have been able to find out the horizontal portion.

After implementing the horizontal projection profile, we have implemented a vertical projection profile which is shown in Figure 4. This is due to the reason when the horizontal projection is used in clipping the horizontal region 
of interest, it includes an extra region both in left and right. To clip those areas, we have used a vertical projection histogram. When the vertical projection is applied upon the clipped horizontal portion, higher peaks will arise for the ending of both sides as it is white. We have considered only the third and fourth highest peaks as for the clipping purpose.

The first and second highest peaks arise because there are two vertical white straight lines. By sorting all four values in descending order we have used the 3rd and 4th highest peak values. By analyzing the peak values, the required re- gion of interest is clipped effectively.

\section{G. Conversion to Binary Image}

Conversion to a binary image is the process of converting a grayscale image into a binary (0-1) image. A binary image has two levels. Binary 1 represents the foreground and binary 0 represents the background. In our project, the reading region needs to be extracted. For that reason, we have converted the gray image into a binary image. But, to convert a grayscale image into a binary image, a good level of threshold value needs to be calculated. This is the main concern for this type of conversion.

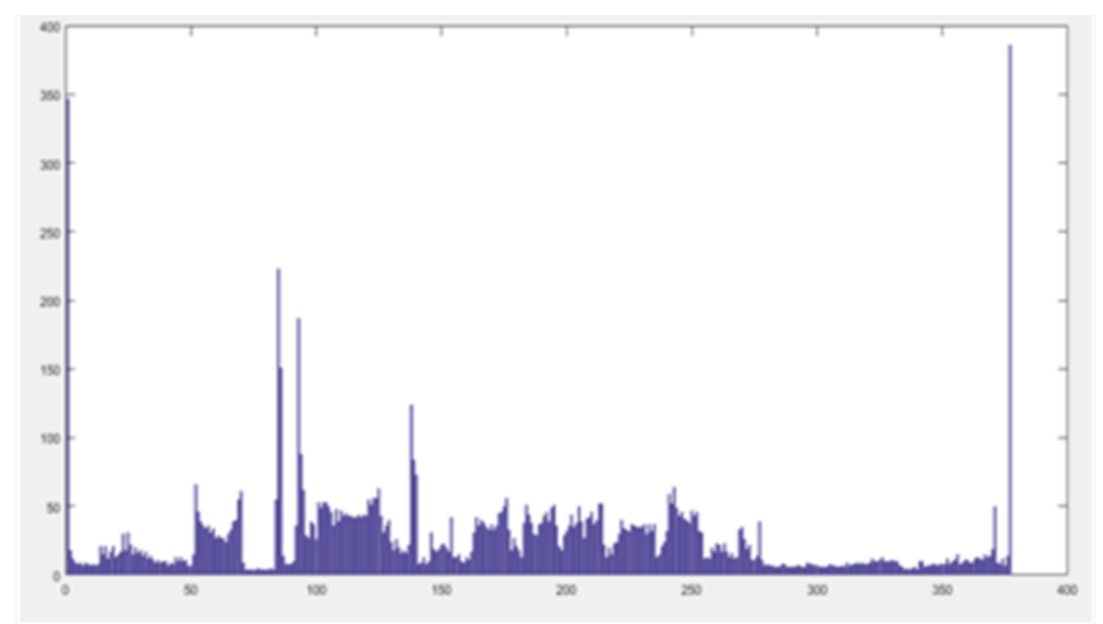

Fig. 5. Horizontal projection profile

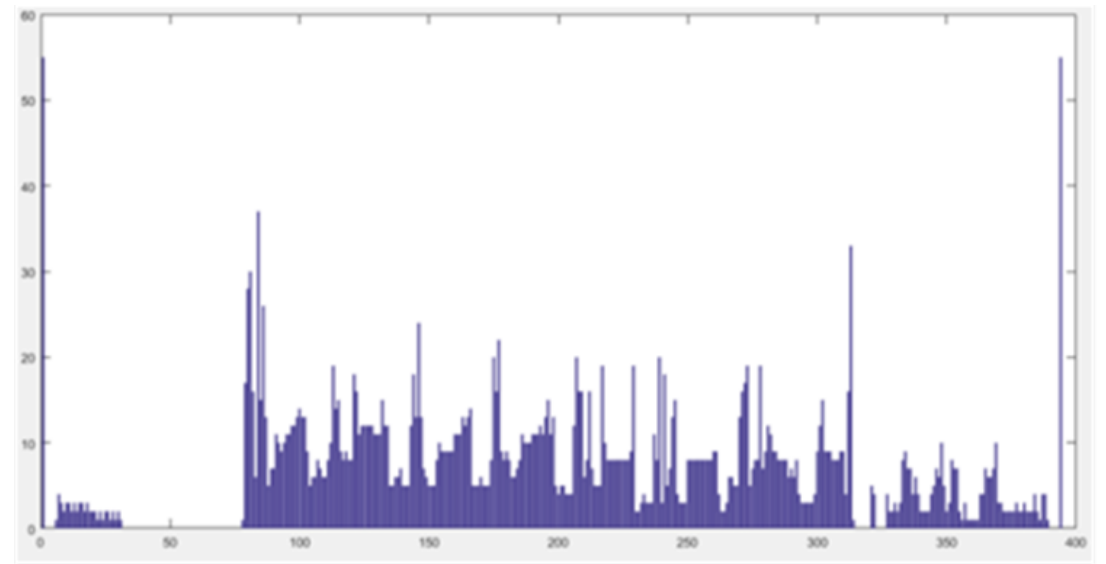

Fig. 6. Vertical projection profile

To avoid manual threshold value, we use the automatic thresholding algorithm which is called the Otsu threshold. This algorithm can be implemented in two ways: withinclass variance and other one is between-class variance. Within-class variance calculates weight, mean, and variance for each intensity level. On the other hand, Between-class variance selects that value having the highest measure of spread. But, between class variance works faster than the within-class variance method.

\section{H. Morphological Operation}

After extracting the region of interest, the segmented digit region consists of non-digit elements in the image. To remove those non-digit elements, the morphological opera- 
tion is performed. To remove this hurdle, we have used the region property toolbox of the MATLAB environment. It removes those non-digit areas by analyzing the minor axis element length and uses the connected component analysis for each digit and removes the element. In a further process, after segmenting the digit image, the individual digits are consumed to get better edges both horizontally and vertically. The segmented digits are of the same width. To process them in the same manner, each digit requires to be shrink. Morphological erosion takes a binary image and places the origin of the structuring element over every pixel position and OR-ing a binary (1) into that position of the output image only if every position of structuring element covers a (1) in the binary image.

\section{Segmentation}

The segmentation of the digit image is categorized by applying the properties of the bounding box algorithm. Each digit area is detected by analyzing, connected component analysis. The digit area is of fixed length. To segment, the digits, vertical projection is used. Generally, most of the water meter reading region consists of six-digit areas. If a vertical projection can be drawn there would be six peaks at a fixed distance. The candidate digit image is then separately segmented through a vertical projection algorithm. A sample segmentation process of a digit image based on a bounding box algorithm is attached in Figure 7.

The segmented digit images are resized to $(12 * 20)$ for further processing. The average accuracy of the segmentation largely depends on the correct use of the bounding box algorithm and the use of vertical projection applications. Having able to segment the water meter digits into the required number of segments and stored the digits in a file for detection and recognition purposes.

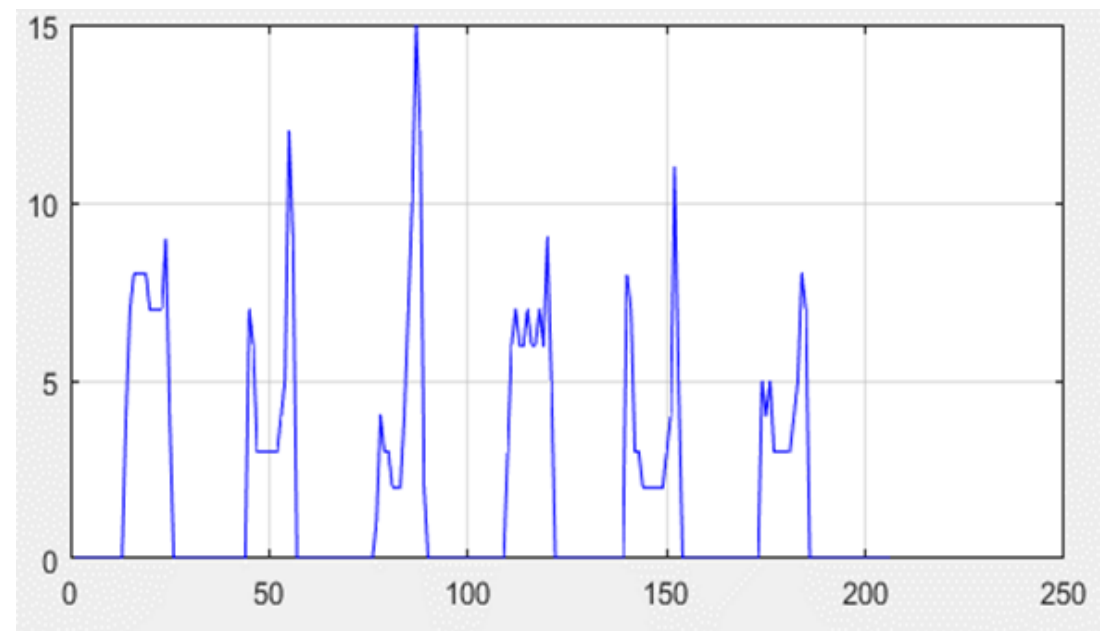

Fig. 7. Bounding box technique for segmentation of a digit image

\section{J. Pattern Generation And Recognition}

To generate the pattern, (0-9) printed digits are taken as sample template images. These template images are used for matching with the digits. Here, for pattern matching, the correlation coefficient is used. The candidate water meter reading image has been recognized based on the value of the correlation coefficient. If the value of the correlation coefficient exceeds a threshold, set by the user, then the input digit image can be assumed to be present in the template datasets and can be effectively recognized. To generate the pattern, (0-9) printed digits are taken as sample template images. These template images are used for matching with the digits. Here, for pattern matching, the correlation coefficient is used. The candidate water meter reading image has been recognized based on the value of the correlation coef- ficient. If the value of the correlation coefficient exceeds a threshold, set by the user, then the input digit image can be assumed to be present in the template datasets and can be effectively recognized.

Here, $A_{m n}$ and $B_{m n}$ are the intensity values of the ith pixel in the first and second images respectively. $\bar{A}$ and $\overline{\mathrm{B}}$ are mean intensity values of the corresponding pixel. When the correlation coefficient has a value equal to 1 , then two images are identical. If the correlation value is equal to 0 , they will be completely uncorrelated. It has the advantages to be invariant to linear transformations of $x$ and $\mathrm{y}$. It also condenses the comparison of two $2-D$ images down to a singular scalar value. The correlation-based pattern recognition 
mathematical expression is given in (4).

$$
r=\frac{\sum_{\mathrm{m}} \Sigma_{\mathrm{n}}\left(\mathrm{A}_{\mathrm{mn}}-\overline{\mathrm{A}}\right)\left(\mathrm{B}_{\mathrm{mn}}-\overline{\mathrm{B}}\right)}{\sqrt{\left(\sum_{\mathrm{m}} \Sigma_{\mathrm{n}}\left(\mathrm{A}_{\mathrm{mn}}-\overline{\mathrm{A}}\right)^{2}\right)\left(\Sigma_{\mathrm{m}} \Sigma_{\mathrm{n}}\left(\mathrm{B}_{\mathrm{mn}}-\overline{\mathrm{B}}\right)^{2}\right)}}
$$

\section{IMPLEMENTATION}

This section will describe the total implementation process of the automation of the water meter reading system and also contain a brief description of the experimental tools used along with our proposed work. We have used the MATLAB environment which is a powerful tool for processing digital images. Moreover, we have implemented our proposed framework for our dataset captured from different houses from Chittagong, Bangladesh.

\section{A. Initial Preprocessing}

All the images are needed to be the same size. To do so, each RGB image in our dataset are resized to $(512 * 512)$ pixels shown in Figure 8.

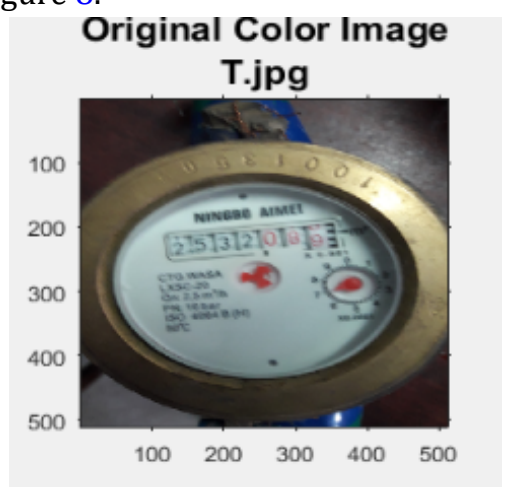

Fig. 8. Bounding box technique for segmentation of a digit image

\section{B. Tilt Correction Technique}

We have used the Hough transformation algorithm to find out the tilted angle and then correct the tilted angle by rotation technique. Two such examples are attached in Figure 9, and Figure 10 for two different samples.

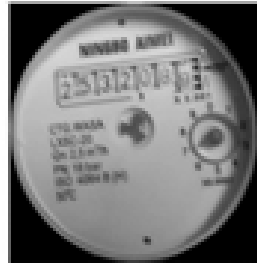

(a)

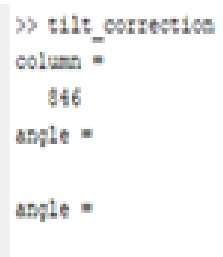

(b)

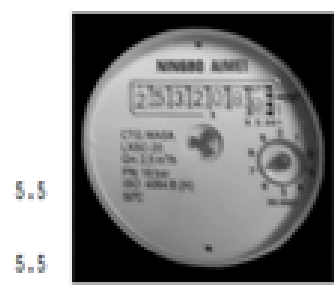

(c)

Fig. 9. Tilt Correction-(I): (a) Tilted meter image, (b) Tilt angle, (c) Corrected meter image

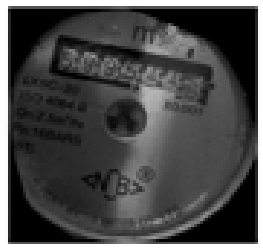

(a)

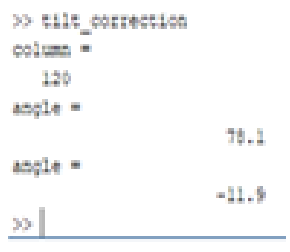

(b)

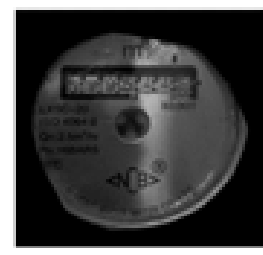

(c)

Fig. 10. Tilt Correction-(II): (a) Tilted meter image, (b) Tilt angle, (c) Corrected meter image

\section{Creating Masked Image}

To generate a mask image, we have converted our RGB image into HSV color space. Using the intensity channel, the meter image is set to the threshold value to create a circular blob shown in Figure 11(b), and (d). Using the largest blob properties, a circular masked image is produced shown in Figure 11 (a), and (c).

The masked meter image contains only the circular shape. Using this masked image, we have to get a rectangular region of interest. But, before proceeding to the next stage, the RGB color image needs to be converted to less computational color space.

\section{Grayscale Masked Image and Enhancement}

The RGB color image is computationally huge costing. To reduce the computational cost and to improve time complexity, it is converted into a grayscale image shown in Figure 12(a). To improve the quality (contrast level), global histogram equalization is applied. The following Figure 12(b) depicts the resultant enhanced image. 


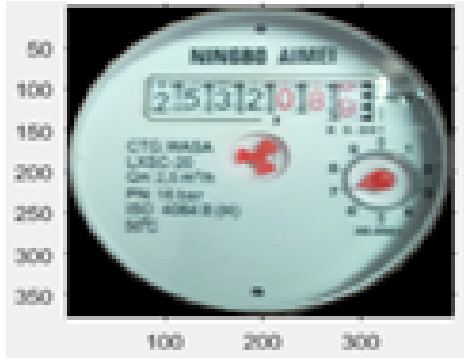

(a)

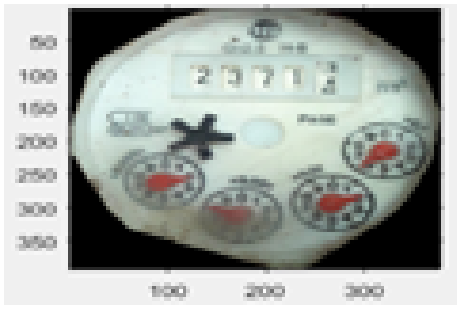

(c)

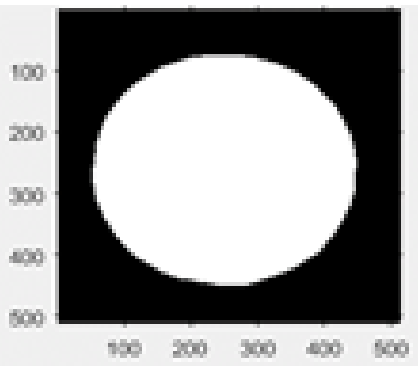

(b)

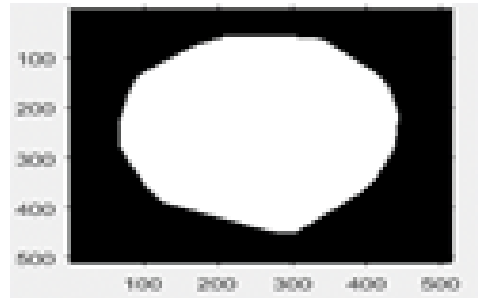

(d)

Fig. 11. (a) Masked image-I, (b)Masking for sample-I, (c) Masked image-II, (d)Masking for sample-II

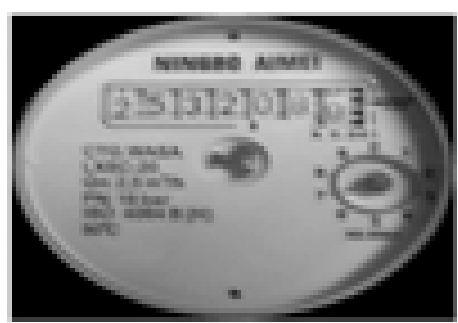

(a)

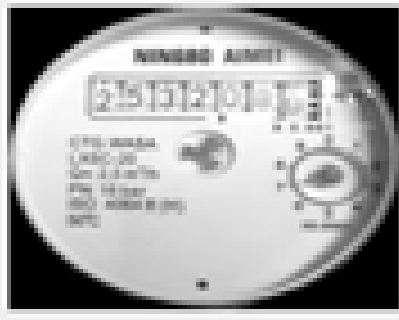

(b)

Fig. 12. (a) Grayscale masked image, (b) Enhanced image

\section{E. Finding Perimeter of the Binary Image}

We have converted the grayscale enhanced image into a binary image. So, in this regard, Otsu thresholding is used to automatically find out the threshold value. The output of the Otsu thresholding is given below in Figure 13(a).

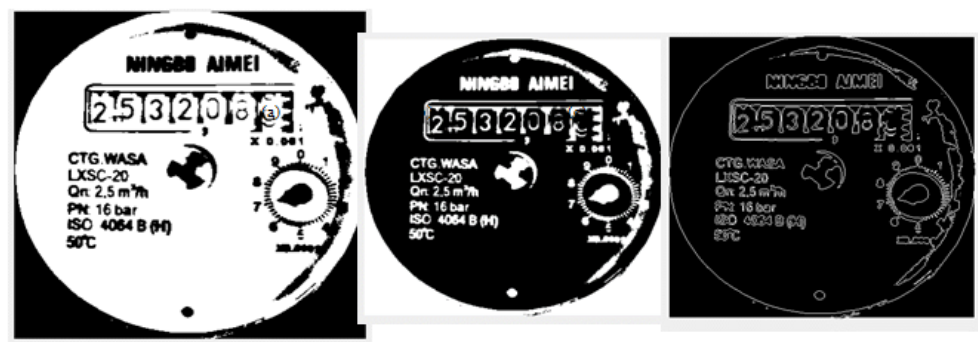

Fig. 13. (a) Otsu thresholding (binary image), (b) Inverse binarization (c) Binary perimeter image

The next stage relates to the inverse of the binary image. This step is necessary because we want our reading area in white pixels. The inverse of binary conversion is shown in Figure 13(b). We need to consider only the perimeter of the previous binary image. The rest of the portion will be binary (0). Using MATLAB, the perimeter of the binary image is turned into binary (1). The above Figure 13(c) illustrates this operation.

\section{F. Finding ROI}

To find the rectangular region of interest, a horizontal projection profile is used. Using third and fourth peak values, 
the horizontal portion is clipped. The resultant figure is shown in the following Figure 14(a), and Figure 14(b). If the vertical projection is drawn upon the horizontal clipped portion, the actual ROI can be extracted. But we have clipped the original binary image not the perimeter image. As the original binary image and the perimeter image are of the same size, the horizontal and vertical projection information can be used as well.

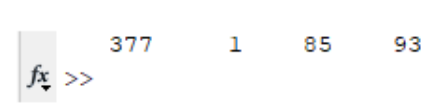

(a)

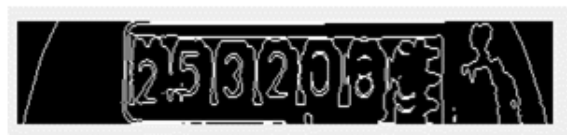

Fig. 14. (a) Horizontal profile peak values,

(b) horizontal clipping

Using third and fourth index values, the vertical portion is clipped. This extracted portion is the region of interest of the project. To get a better result in the following parts, a morphological filling algorithm is applied to the extracted final ROI shown in Figure 15 (a), and Figure 15 (b).

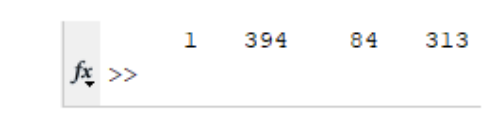

(a)

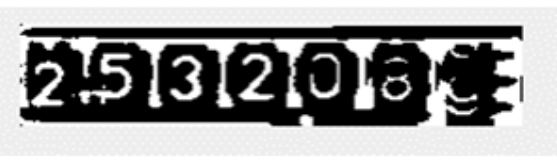

(b)

Fig. 15. (a) Vertical profile peak values, (b) Final extracted ROI

\section{G. Noise Reduction}

To reduce the noise from the ROI image, a morphological operation is performed shown in Figure 16(a). This technique successfully gives the result a noise-free digit image. This digit image is then further processed for the next stage. Morphological erosion is performed to produce a thin digit image. The following Figure 16(b) illustrates a noise reduction of the digit image and the result of the morphological erosion.

\section{8}

(a)

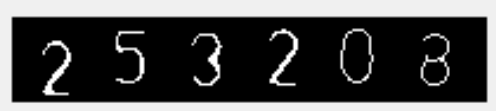

(b)

Fig. 16. (a) Noise reduction, (b) Morphological erosion

\section{H. Segmentation of the Digit Image}

To segment the digit image into individual digits, the selection of each area is done by a bounding box algorithm. The following Figure 17 shows each digit area by a rectangular red shape. The segmented individual digits are stored in a file for further process.

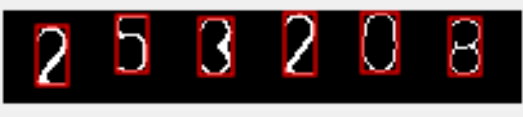

(a)

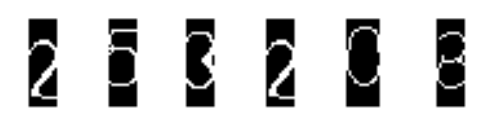

(b)

Fig. 17. (a) Implementation of the bounding box, (b) Segmentation of digit image (high illumination)

\section{Recognition by Correlation Coefficient}

Using correlation coefficient values, best-matched digits are shown. After segmenting the digit image, segmented digits are passed to the recognition system. By analyzing the similarities of Normalized Cross-Correlation (NCC) values, the reading region can be recognized. The whole process is illustrated in Figure 18.

\section{EXPERIMENTAL RESULTS}

The following Table 1 will analyze the results of our performed experiment in different environmental conditions and Table 2 will give a comparison between our proposed framework with [3]. The following graphical representation shown in Figure 19 will help to understand briefly the significance of our proposed framework for extracting meter readings values and hence to prepare water bills automatically. Besides, we have added some sample datasets upon which we have applied our proposed method to get the results are shown in Figure 20 and Figure 21 for different environmental conditions. 
TABLE 1

THE RESULT OBTAINED FROM DIFFERENT ENVIRONMENTS

\begin{tabular}{|c|c|c|c|c|}
\hline Environmental Condition & Number of Meter Images & $\begin{array}{l}\text { Correctly Detected Reading } \\
\text { Region }\end{array}$ & $\begin{array}{l}\text { Correctly Segmented Reading Re- } \\
\text { gion }\end{array}$ & $\begin{array}{l}\text { Correctly Recognized } \\
\text { Readings }\end{array}$ \\
\hline Properly illuminated & 150 & 150 & 147 & 147 \\
\hline Varying illumination & 50 & 49 & 48 & 48 \\
\hline Under noisy condition & 30 & 27 & 27 & 26 \\
\hline Total & 230 & 226 & 222 & 221 \\
\hline
\end{tabular}

TABLE 2

COMPARISON OF ACCURACY RATE

\begin{tabular}{llll}
\hline \hline & Number of Meter Images & Correctly Recognized & Success Rate (\%) \\
\hline$[3]$ & 135 & 127 & 94.1 \\
Proposed Framework & 230 & 221 & 96 \\
\hline \hline
\end{tabular}

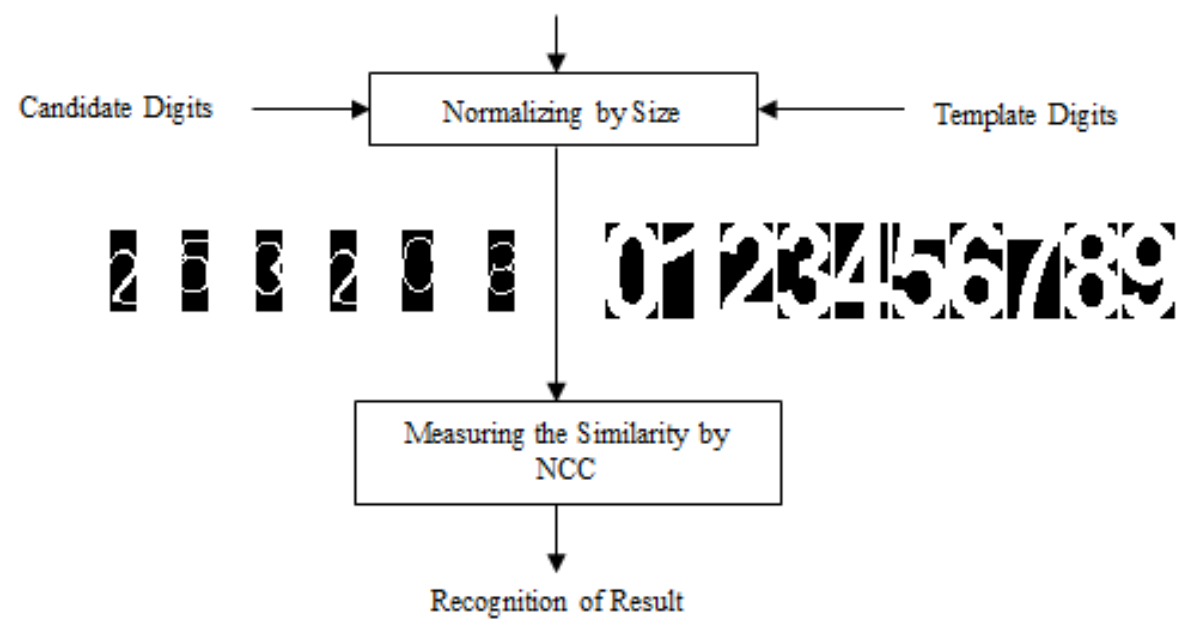

Fig. 18. Water meter reading recognition schema

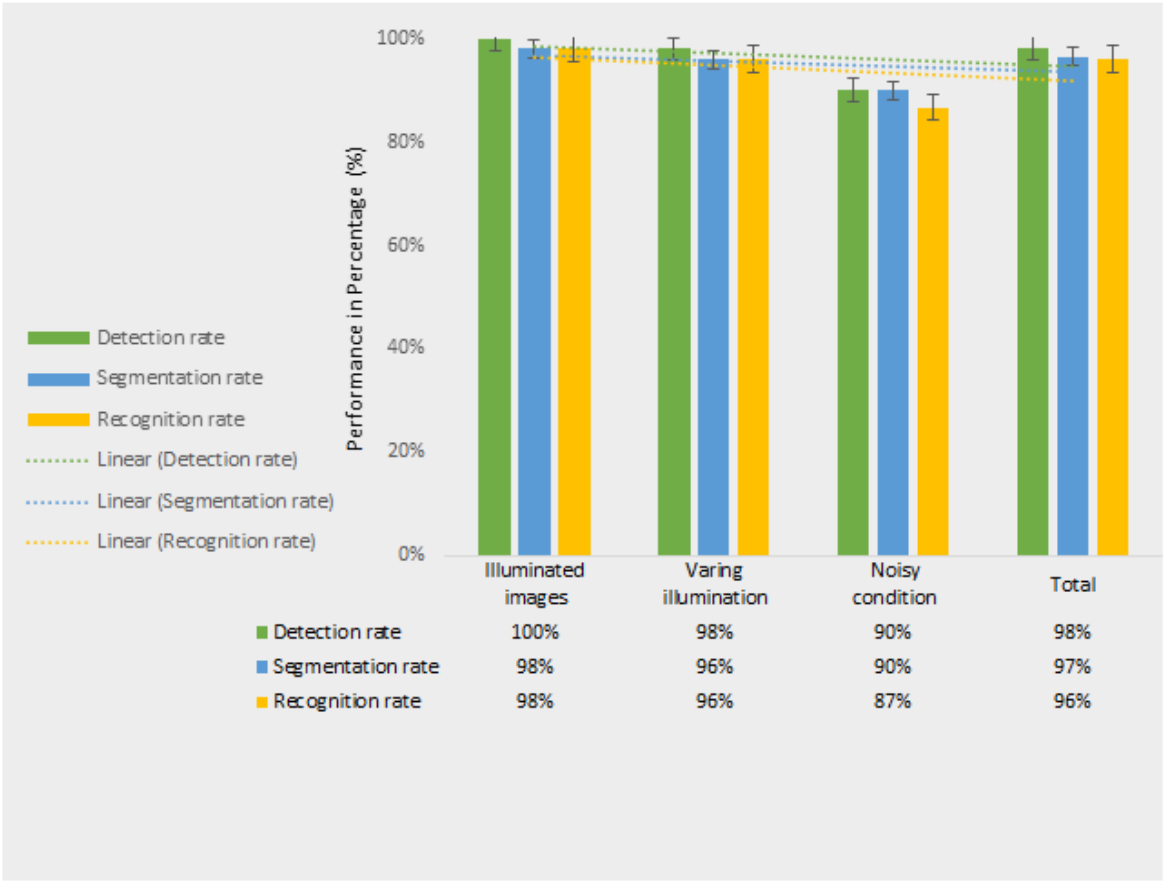

Fig. 19. Performance analysis graph 


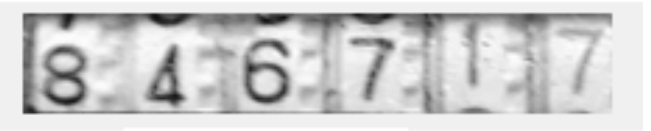

(a)

\subsection{7}

(b)

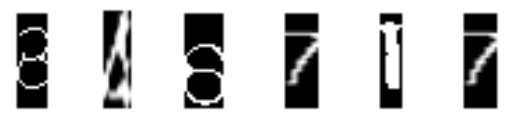

(c)

Fig. 20. (a) Digit image in Grayscale, (b) Converted to a binary image, (c) Segmented digits from the image (varying illumination)

\section{$5: 9: 9 ; 5: 4: 4$}

(a)

\section{4}

(b)

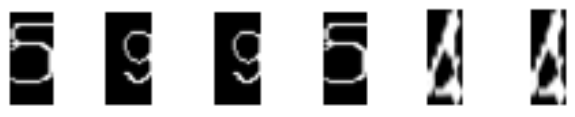

(c)

Fig. 21. (a) Digit image in Grayscale, (b) Converted to a binary image, (c) Segmented digits from the image (noisy condition)

\section{CONCLUSION}

In this paper, correlation coefficient-based water meter readings recognition is proposed. We have gained (96\%) overall accuracy to extract meter readings from the meter images. We have implemented a horizontal and vertical projection profile algorithm to get our desired region of interest region from the meter images. The extracted region of interest is then normalized by the morphological operation. Segmentation is performed by horizontal projection. The segmented digits are matched with the template digits by comparing correlation coefficient values. Accurate extraction of the reading region is mostly dependent on finding a good quality masked image. Removing noises from the extracted region is important for segmentation. Well, segmented digits help to improve the success rate. If noise from the binary image can be removed totally, the recognition rate can be increased. This work has been accomplished for the automation of the water meter reading process and hence to apply in the automation of the billing process.

This paper depicts only the automation of the water meter reading process and hence to generate bills automatically. In the future, our proposed framework can be integrated with a real-time monitoring system that can identify water pipe leakage problems and can identify excess use of water consumption limits for ensuring a better centrally water management system. 


\section{REFERENCES}

[1] Z. Zhang and Y. Li, "Research on the pre-processing method of automatic reading water meter system," in International Conference on Artificial Intelligence and Computational Intelligence, California, CA, 2009.

[2] S. G. Kashid and S. A. Pardeshi, "Intelligent water metering system: An image processing approach (matlab simulations)," in First International Conference on Networks \& Soft Computing (ICNSC2014), New York, NY, 2014.

[3] A. Anis, M. Khaliluzzaman, M. Yakub, N. Chakraborty, and K. Deb, "Digital electric meter reading recognition based on horizontal and vertical binary pattern," in 3rd International Conference on Electrical Information and Communication Technology (EICT), Bangkok, Thailand, 2017.

[4] B. R. Koh, Y. Hun Oh, and D. Park, "A study on formation of saltwater-freshwater interface and upconing in coastal aquifers based on experiments with hydraulic models," Journal of Advances in Technology and Engineering Studies, vol. 2, no. 5, pp. 134-139, 2016. doi: Ihttps://dx.doi.org/10.20469/ijtes.3.40002-5

[5] V. C. P. Edward, "Support vector machine based automatic electric meter reading system," in IEEE International Conference on Computational Intelligence and Computing Research, New Dehli, India, 2013.

[6] S. Sarkar and V. Ilango, "Improvement of fuel cell performance by application of carbon nanotubes," International Journal of Technology and Engineering Studies, vol. 2, no. 6, pp. 180-184, 2016. doi: https://doi.org/10.20469/ijtes.2. 40003-6

[7] L. A. Elrefaei, A. Bajaber, S. Natheir, N. AbuSanab, and M. Bazi, “Automatic electricity meter reading based on image processing," in IEEE Jordan Conference on Applied Electrical Engineering and Computing Technologies (AEECT), Kulalumpur, Malaysia, 2015.

[8] D. Shu, S. Ma, and C. Jing, "Study of the automatic reading of watt meter based on image processing technology," in 2nd IEEE Conference on Industrial Electronics and Applications, London, UK, 2007.

[9] Y. Zhang, S. Yang, X. Su, E. Shi, and H. Zhang, "Automatic reading of domestic electric meter: An intelligent device based on image processing and ZigBee/Ethernet communication," Journal of Real-Time Image Processing, vol. 12, no. 1, pp. 133-143, 2016. doi: https://doi.org/10.1007/s11554-013-0361-2

[10] K. Parthiban and A. Palanisamy, "Reading values in electrical meter using image processing techniques," in International Conference on Intelligent Interactive Systems and Assistive Technologies, New Jersy, NJ, 2013.

[11] W. Guolu, Q. Kaijin, N. Tongxin, and X. Guoqiang, "The design and implementation of an ad hoc-based wireless meter reading system," in IEEE 4th International Conference on Software Engineering and Service Science, New York, NY, 2013.

[12] K. Kanayama, Y. Fujikawa, K. Fujimoto, and M. Horino, "Development of vehicle-license number recognition system using real-time image processing and its application to travel-time measurement," in Proceedings 41st IEEE Vehicular Technology Conference, Florida, FL, 1991.

[13] E. Tekin, J. M. Coughlan, and H. Shen, "Real-time detection and reading of led/lcd displays for visually impaired persons," in IEEE Workshop on Applications of Computer Vision (WACV), California, CA, 2011. 